\title{
A Time-Splitting and Sine Spectral Method for Dynamics of Dipolar Bose-Einstein Condensate
}

\author{
Si-Qi Li, Xiang-Gui Li, and Dong-Ying Hua \\ School of Applied Science, Beijing Information Science and Technology University, Beijing 100192, China \\ Correspondence should be addressed to Xiang-Gui Li; xianggui-li@vip.sina.com
}

Received 28 May 2013; Accepted 9 July 2013

Academic Editor: Fedele Lizzi

Copyright (C) 2013 Si-Qi Li et al. This is an open access article distributed under the Creative Commons Attribution License, which permits unrestricted use, distribution, and reproduction in any medium, provided the original work is properly cited.

\begin{abstract}
A two-component Bose-Einstein condensate (BEC) described by two coupled a three-dimension Gross-Pitaevskii (GP) equations is considered, where one equation has dipole-dipole interaction while the other one has only the usual s-wave contact interaction, in a cigar trap. The time-splitting and sine spectral method in space is proposed to discretize the time-dependent equations for computing the dynamics of dipolar BEC. The singularity in the dipole-dipole interaction brings significant difficulties both in mathematical analysis and in numerical simulations. Numerical results are given to show the efficiency of this method.
\end{abstract}

\section{Introduction}

The achievement of the Bose-Einstein condensation of dilute gases in 1995 marked the beginning of a new era in atomic, molecular, and optical physics. That has attracted much attention both theoretically and experimentally. Most of their properties of these trapped quantum gases are governed by the interactions between particles in the condensate [1]. In the last several years, there has been an investigation for realizing a new kind of quantum gases with the dipolar interaction, acting between particles having a permanent magnetic or electric dipole moment. The experimental realization of a $\mathrm{BEC}$ of 52Cr atoms [2,3] at the University of Stuttgart in 2005 gave new impetus to the theoretical and the numerical investigations on these novel dipolar quantum gases at low temperature. Recently more detailed and controlled experimental results have been obtained, illustrating the effects of phase separation in a multicomponent BEC [4-6]. In these papers, the studies of the binary condensates were limited to the case of s-wave interaction, while a great deal of attention has been drawn recently to the dipolar BEC.

In this work, a numerical method for computing the dynamics of the two-component dipolar BEC is considered, where one equation has dipole-dipole interaction and the other has only the usual s-wave contact interaction. However, since the dipole-dipole interaction is of long range, anisotropic, and partially attractive and the computational cost in three dimensions high, the nontrivial task of achieving and controlling the dipolar BEC is thus particularly challenging.

This paper is organized as follows. In Section 2, a numerical method for computing ground states is presented. In Section 3, numerical results are reported to verify the efficiency of this numerical method. Finally, some concluding remarks are drawn in Section 4.

\section{Numerical Method for Computing the Dynamics}

2.1. The Nonlocal Gross-Pitaevskii Equation. The two-component dipolar BEC, confined in a cigar trap, is described by two coupled Gross-Pitaevskii equations. As far as the dipolar interaction is concerned, a convolution term is introduced [7-9] to modify the classical Gross-Pitaevskii equation, which results in the following differential-integral equations (1). Since the transition metal has a magnetic dipole interaction while the alkali metal does not have, we take into account this factor in this system. We take $\mathrm{Cr}$ as component 1 and $\mathrm{Rb}$ as component 2 [10]. Then the GP equations for this system can be written as

$$
\begin{gathered}
i \hbar \frac{\partial \varphi_{1}(\vec{r}, t)}{\partial t}=\left[-\frac{\hbar^{2}}{2 m_{1}} \nabla^{2}+V_{1}+U_{1}\left|\varphi_{1}\right|^{2}+U_{12}\left|\varphi_{2}\right|^{2}\right. \\
\left.+V_{\text {dip }} *\left|\varphi_{1}\right|^{2}\right] \varphi_{1}
\end{gathered}
$$




$$
i \hbar \frac{\partial \varphi_{2}(\vec{r}, t)}{\partial t}=\left[-\frac{\hbar^{2}}{2 m_{2}} \nabla^{2}+V_{2}+U_{2}\left|\varphi_{2}\right|^{2}+U_{12}\left|\varphi_{1}\right|^{2}\right] \varphi_{2},
$$

where $\varphi_{1}, \varphi_{2}$ are the wave functions of components one and two, respectively. The interatomic and the intercomponent swave scattering interactions are described by $U_{j}(j=1,2)$ and $U_{12}$, respectively, with the following expressions [11]:

$$
U_{j}=\frac{4 \pi \hbar^{2} a_{j}}{m_{j}}, \quad U_{12}=\frac{2 \pi \hbar^{2} a_{j}}{m_{1} m_{2} /\left(m_{1}+m_{2}\right)}, \quad j=1,2,
$$

where $a_{j}$ is the scattering length of component $j$ and $a_{12}$ is that between components 1 and 2 . Here $\hbar$ is the Planck constant, $m_{j}$ is the mass of the atom of component $j, j=1,2$, and $V_{j}$ is the external trapping potential confining the gas. Generally, that is, $V_{j}(\vec{r})=\left(m_{j} / 2\right)\left(\omega_{j x}^{2} x^{2}+\omega_{j y}^{2} y^{2}+\omega_{j z}^{2} z^{2}\right)$ with $\omega_{j p}(p=x, y, z)$ representing the trap frequency in $x, y$, and $z$ directions, respectively. The local mean-field $U_{j}\left|\varphi_{j}\right|^{2}$ represents the s-wave interaction. $V_{\text {dip }}$ is the longrange isotropic dipolar interaction potential between two dipoles and it is defined by

$$
V_{\mathrm{dip}}=\frac{\mu_{0} \mu_{\mathrm{dip}}^{2}}{4 \pi} \cdot \frac{1-3(\vec{r} \cdot \vec{n})^{2} /|\vec{r}|^{2}}{|\vec{r}|^{3}}=\frac{\mu_{0} \mu_{\mathrm{dip}}^{2}}{4 \pi} \cdot \frac{1-3 \cos ^{2} \theta}{|\vec{r}|^{3}},
$$

where $\theta$ is the angle between the polarization axis $\vec{n}$ and the relative of two atoms (i.e., $\cos \theta=\vec{n} \cdot \vec{r} / r, r=|\vec{r}|=$ $\left.\sqrt{x^{2}+y^{2}+z^{2}}\right)$. The wave function $\varphi_{i}(x, t)$ is normalized according to $\left\|\varphi_{i}\right\|^{2}=\int_{R^{3}}\left|\varphi_{i}(\vec{r}, t)\right|^{2} d \vec{r}=N_{i}, i=1,2$, where $N_{i}$ is the number of the atoms in the dipolar BEC.

\subsection{Time-Splitting and Sine Spectral Numerical Method for} Dynamics. The system (1) can be made dimensionless and simplified by adopting a unit system where the units for length, time, and energy are given by $a_{0}, 1 / \omega_{0}$, and $\hbar \omega_{0}$, respectively, with $\omega_{0}=\min \left\{\omega_{j x}, \omega_{j y}, \omega_{j z}\right\}, a_{0}=\sqrt{\hbar / m_{1} \omega_{0}}[12]$.

By introducing the dimensionless variables $t^{\prime}=t / \omega_{0}$, $\vec{r}^{\prime}=\vec{r} / a_{0}, \varphi_{j}^{\prime}=a_{0}^{3 / 2} \varphi_{j}$, we obtain the dimensionless GP equations in $3 \mathrm{D}$ from (1) as follows:

$$
\begin{gathered}
i \frac{\partial \varphi_{1}^{\prime}}{\partial t^{\prime}}=\left[-\frac{1}{2} \nabla^{2}+V_{1}^{\prime}(\vec{r})+\beta_{11}\left|\varphi_{1}^{\prime}\right|^{2}\right. \\
\left.+\beta_{12}\left|\varphi_{2}^{\prime}\right|^{2}+\lambda\left(V_{\mathrm{dip}} *\left|\varphi_{1}^{\prime}\right|^{2}\right)\right] \varphi_{1}^{\prime}, \\
i \frac{\partial \varphi_{2}^{\prime}}{\partial t^{\prime}}=\left[-\frac{1}{2} \nabla^{2}+a_{m} V_{2}^{\prime}(\vec{r})+\beta_{21}\left|\varphi_{1}^{\prime}\right|^{2}+\beta_{22}\left|\varphi_{2}^{\prime}\right|^{2}\right] \varphi_{2}^{\prime},
\end{gathered}
$$

where $\beta_{11}=4 \pi a_{1} N_{1}, \beta_{12}=\left(\left(1+a_{m}\right) / a_{m}\right) 2 \pi a_{12} N_{2}, \beta_{21}=$ $\left(\left(1+a_{m}\right) / a_{m}\right) 2 \pi a_{12} N_{1}, \beta_{22}=\left(4 \pi a_{2} / a_{m}\right) N_{2}, a_{m}=m_{2} / m_{1}$, $\lambda=m_{1} N_{1} \mu_{0} \mu_{\mathrm{dip}}^{2} / 3 \hbar^{2} a_{0}$, and $V_{\mathrm{dip}}=(3 / 4 \pi) \cdot\left(\left(1-3 \cos ^{2} \theta\right) / r^{3}\right)$.
In addition the wave functions in (4) satisfy $\int_{R^{3}}\left|\varphi_{1}^{\prime}\right|^{2}=1$, $\int_{R^{3}}\left|\varphi_{2}^{\prime}\right|^{2}=1$. By using the following formula [13]

$$
\frac{1}{r^{3}}\left(1-\frac{3(\vec{n} \cdot \vec{r})^{2}}{r^{2}}\right)=-\frac{3}{4 \pi} \delta(\vec{r})-\partial_{n n}\left(\frac{1}{r}\right),
$$

where $\delta(\vec{r})$ is the Dirac delta function and $\partial_{n n}=\vec{n} \cdot \nabla(\vec{n} \cdot \nabla)$, we can get

$$
V_{\mathrm{dip}} *\left|\varphi_{1}^{\prime}\right|^{2}=-\left|\varphi_{1}^{\prime}\right|^{2}-3 \partial_{n n}(\xi)
$$

where

$$
\xi(\vec{r}, t)=\frac{1}{4 \pi} \int_{R^{3}} \frac{1}{\left|\vec{r}-\vec{r}^{\prime}\right|} \cdot\left|\varphi_{1}^{\prime}\left(\vec{r}^{\prime}, t\right)\right|^{2} d \vec{r}^{\prime} .
$$

And it is easy to see that

$$
\nabla^{2} \xi=-\left|\varphi_{1}^{\prime}(\vec{r}, t)\right|^{2}
$$

Plugging (6) into (4) and noticing (7) and (8), we can reformulate GPE (4) into the Schrödinger-Poisson type system

$$
\begin{gathered}
i \frac{\partial \varphi_{1}}{\partial t}=\left[-\frac{1}{2} \nabla^{2}+V_{1}+\left(\beta_{11}-\lambda\right)\left|\varphi_{1}\right|^{2}\right. \\
\left.+\beta_{12}\left|\varphi_{2}\right|^{2}-3 \lambda \partial_{n n}(\xi)\right] \varphi_{1}, \\
i \frac{\partial \varphi_{2}}{\partial t}=\left[-\frac{1}{2} \nabla^{2}+V_{2}+\beta_{21}\left|\varphi_{1}\right|^{2}+\beta_{22}\left|\varphi_{2}\right|^{2}\right] \varphi_{2}, \\
\nabla^{2} \xi=-\left|\varphi_{1}(\vec{r}, t)\right|^{2} .
\end{gathered}
$$

In practice, the whole space problem is usually truncated into a bounded computational domain $\Omega=[a, b] \times[c, d] \times[e, f]$ with the homogeneous Dirichlet boundary condition. Let

$$
\begin{gathered}
\Omega_{M K L}=\{(j, k, l) \mid j=1,2, \ldots, M-1, k=1,2, \\
\ldots, K-1, l=1,2, \ldots, L-1\}, \\
\Omega_{M K L}^{0}\{(j, k, l) \mid j=0,1, \ldots, M, k=0,1, \ldots, K, \\
l=0,1, \ldots, L\} .
\end{gathered}
$$

Choose the spatial mesh size as $h_{x}=(b-a) / M, h_{y}=(d-c) / K$ and $h_{z}=(f-e) / L$ and define $x_{j}=a+j h_{x}, y_{k}=c+k h_{y}$, and $z_{l}=e+l h_{z}, j, k, l \in \Omega_{M K L}^{0}$. Then denote the space $Y_{M K L}=$ $\operatorname{span}\left\{\phi_{p q s}(\vec{r}),(p, q, s) \in \Omega_{M K L}\right\}$ with

$$
\phi_{p q s}(r)=\sin \left(u_{p}(x-a)\right) \sin \left(u_{q}(y-c)\right) \sin \left(u_{s}(z-e)\right) \text {, }
$$

where $u_{p}=p \pi /(b-a), u_{q}=q \pi /(d-c)$, and $u_{s}=s \pi /(f-e)$.

We propose a time-splitting sine pseudo-spectral method for computing the dynamics of the BEC [12]. 
From $t=t_{k}$ to $=t_{k+1}$, the GP equation (9) is solved by three steps. First, we solve

$$
\begin{gathered}
i \frac{\partial \varphi_{1}(\vec{r}, t)}{\partial t}=-\frac{1}{2} \nabla^{2} \varphi_{1}(\vec{r}, t), \\
i \frac{\partial \varphi_{2}(\vec{r}, t)}{\partial t}=-\frac{1}{2} \nabla^{2} \varphi_{2}(\vec{r}, t), \\
\left.\varphi_{1}(\vec{r}, t)\right|_{r \in \partial \Omega}=0,\left.\quad \varphi_{2}(\vec{r}, t)\right|_{r \in \partial \Omega}=0
\end{gathered}
$$

from $t_{k}$ to $t_{k+1 / 2}$, followed by solving the nonlinear ODE

$$
\begin{gathered}
i \frac{\partial \varphi_{1}}{\partial t}=\left[V_{1}+\left(\beta_{11}-\lambda\right)\left|\varphi_{1}\right|^{2}+\beta_{12}\left|\varphi_{2}\right|^{2}-3 \lambda \partial_{n n}(\xi)\right] \varphi_{1}, \\
i \frac{\partial \varphi_{2}}{\partial t}=\left[V_{2}+\beta_{21}\left|\varphi_{1}\right|^{2}+\beta_{22}\left|\varphi_{2}\right|^{2}\right] \varphi_{2}, \\
\nabla^{2} \xi=-\left|\varphi_{1}(\vec{r}, t)\right|^{2}, \\
\left.\varphi_{1}(\vec{r}, t)\right|_{r \in \partial \Omega}=0,\left.\quad \varphi_{2}(\vec{r}, t)\right|_{r \in \partial \Omega}=0, \\
\vec{r} \in \Omega t_{n} \leq t \leq t_{n+1}
\end{gathered}
$$

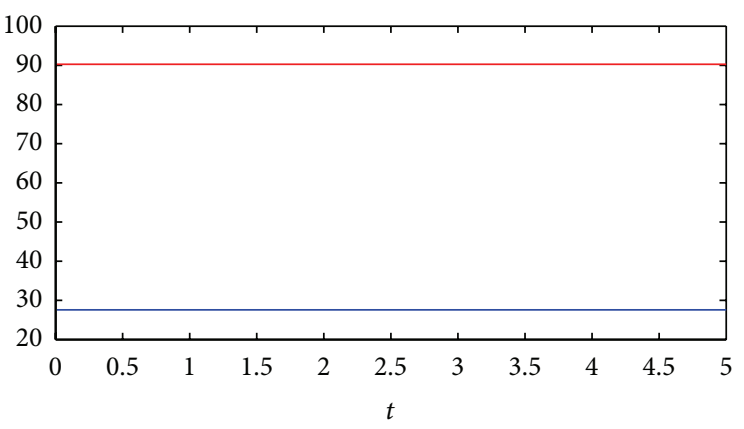

$-\varphi_{1}$
$-\varphi_{2}$

FIGURE 1: The energy evolution according to $t$.

$$
\begin{gathered}
\times \alpha_{p q s}^{\prime} \sin \frac{j p \pi}{M} \sin \frac{k q \pi}{K} \sin \frac{l s \pi}{L}, \\
(j, k, l) \in \Omega_{M K L}^{0},
\end{gathered}
$$

(13) where

for one time step. Again, we solve (12) from $t_{k+1 / 2}$ to $t_{k+1}$.

Suppose the exact solutions are

$$
\begin{aligned}
& \varphi_{1}\left(x_{j}, y_{k}, z_{l}, t_{n}\right) \\
& =\varphi_{1 j k l}^{n}=\sum_{p=1}^{M-1} \sum_{q=1}^{K-1} \sum_{s=1}^{L-1} \varphi_{1 p q s}\left(t_{n}\right) \sin \frac{j p \pi}{M} \sin \frac{k q \pi}{K} \sin \frac{l s \pi}{L},
\end{aligned}
$$

$$
\begin{aligned}
& \varphi_{2}\left(x_{j}, y_{k}, z_{l}, t_{n}\right) \\
& =\varphi_{2 j k l}^{n}=\sum_{p=1}^{M-1} \sum_{q=1}^{K-1} \sum_{s=1}^{L-1} \varphi_{2 p q s}\left(t_{n}\right) \sin \frac{j p \pi}{M} \sin \frac{k q \pi}{K} \sin \frac{l s \pi}{L} .
\end{aligned}
$$

Substitute (14), (15) into (12); we can find that

$$
\begin{array}{r}
i \frac{d}{d t} \varphi_{1 p q s}\left(t_{n}\right)=\frac{u_{p}^{2}+u_{q}^{2}+u_{s}^{2}}{2} \varphi_{1 p q s}\left(t_{n}\right), \\
i \frac{d}{d t} \varphi_{2 p q s}\left(t_{n}\right)=\frac{u_{p}^{2}+u_{q}^{2}+u_{s}^{2}}{2} \varphi_{2 p q s}\left(t_{n}\right), \\
(p, q, s) \in \Omega_{M K L}^{0},
\end{array}
$$

which can be solved exactly, and we obtain

$$
\begin{aligned}
& \varphi_{1 j k l}^{(1)}=\sum_{p=1}^{M-1} \sum_{q=1}^{K-1} \sum_{s=1}^{L-1} e^{-i \tau\left(\left(u_{p}^{2}+u_{q}^{2}+u_{s}^{2}\right) / 4\right)} \\
& \quad \times \alpha_{p q s} \sin \frac{j p \pi}{M} \sin \frac{k q \pi}{K} \sin \frac{l s \pi}{L}, \\
& \varphi_{2 j k l}^{(1)}=\sum_{p=1}^{M-1} \sum_{q=1}^{K-1} \sum_{s=1}^{L-1} e^{-i \tau\left(\left(u_{p}^{2}+u_{q}^{2}+u_{s}^{2}\right) / 4\right)}
\end{aligned}
$$

$$
\begin{aligned}
& \alpha_{p q s}=\frac{8}{M K L} \sum_{p q s} \varphi_{1 j k l}^{n} \sin \frac{j p \pi}{M} \sin \frac{k q \pi}{K} \sin \frac{l s \pi}{L}, \\
& \alpha_{p q s}^{\prime}=\frac{8}{M K L} \sum_{p q s} \varphi_{2 j k l}^{n} \sin \frac{j p \pi}{M} \sin \frac{k q \pi}{K} \sin \frac{l s \pi}{L} .
\end{aligned}
$$

Equations (12) will be discretized in space by sine pseudospectral method and integrated in time [14]. Next, we will show that (13) can be solved exactly.

In fact, for $t \in\left[t_{n}, t_{n+1}\right]$, multiplying (13) by the conjugation of $\varphi(r, t)$, that is, $\overline{\varphi(r, t)}$, we get

$$
\begin{gathered}
i \frac{\partial \varphi_{1}}{\partial t} \overline{\varphi_{1}}=\left[V_{1}+\left(\beta_{11}-\lambda\right)\left|\varphi_{1}\right|^{2}+\beta_{12}\left|\varphi_{2}\right|^{2}\right. \\
\left.-3 \lambda \partial_{n n}(\xi)\right] \varphi_{1} \overline{\varphi_{1}}
\end{gathered}
$$

$$
i \frac{\partial \varphi_{2}}{\partial t} \overline{\varphi_{2}}=\left[V_{2}+\beta_{21}\left|\varphi_{1}\right|^{2}+\beta_{22}\left|\varphi_{2}\right|^{2}\right] \varphi_{2} \overline{\varphi_{2}},
$$

and we also have

$$
\begin{gathered}
-i \frac{\partial \overline{\varphi_{1}}}{\partial t} \varphi_{1}=\left[V_{1}+\left(\beta_{11}-\lambda\right)\left|\varphi_{1}\right|^{2}+\beta_{12}\left|\varphi_{2}\right|^{2}\right. \\
\left.-3 \lambda \partial_{n n}(\xi)\right] \overline{\varphi_{1}} \varphi_{1}, \\
-i \frac{\partial \overline{\varphi_{2}}}{\partial t} \varphi_{2}=\left[V_{2}+\beta_{21}\left|\varphi_{1}\right|^{2}+\beta_{22}\left|\varphi_{2}\right|^{2}\right] \overline{\varphi_{2}} \varphi_{2} .
\end{gathered}
$$

Therefore, subtracting (13) from (14), one obtains

$$
i \frac{d\left|\varphi_{i}(\vec{r}, t)\right|^{2}}{d t}=0, \quad i=1,2,
$$

which implies that

$$
\left|\varphi_{i}(\vec{r}, t)\right|^{2}=\left|\varphi_{i}\left(\vec{r}, t_{k}\right)\right|^{2}, \quad i=1,2 \forall k, t_{k} \leq t \leq t_{k+1} .
$$



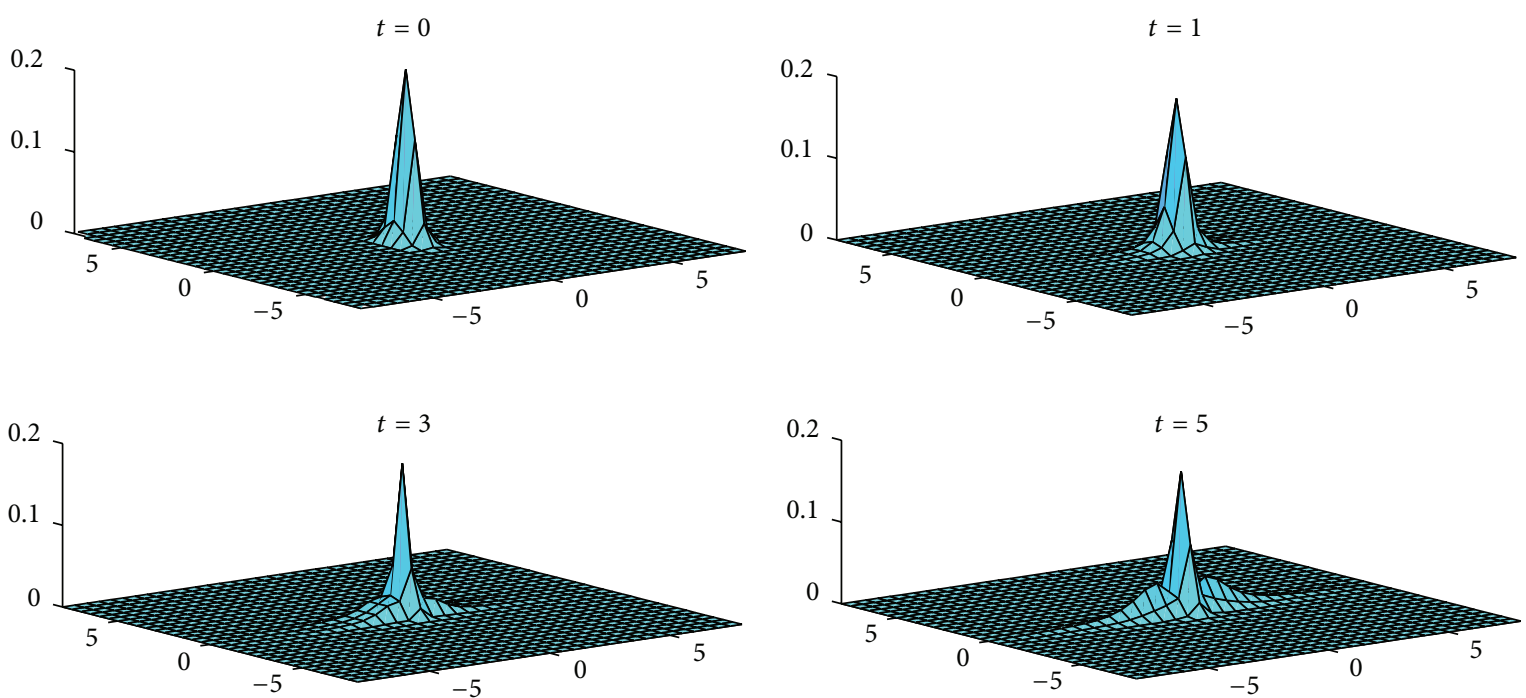

Figure 2: The wave function evolution according to time. Surface plots for $\left|\varphi_{1}(x, 0, z, t)\right|^{2}$ at different times.

Substituting (22) into (13), we get a linear ODE

$$
\begin{gathered}
i \frac{\partial \varphi_{1}(\vec{r}, t)}{\partial t}=\left[V_{1}+\left(\beta_{11}-\lambda\right)\left|\varphi_{1}\left(\vec{r}, t_{k}\right)\right|^{2}\right. \\
\left.+\beta_{12}\left|\varphi_{2}\left(\vec{r}, t_{k}\right)\right|^{2}-3 \lambda \partial_{n n}(\xi)\right] \varphi_{1}(\vec{r}, t) \\
i \frac{\partial \varphi_{2}(\vec{r}, t)}{\partial t}=\left[V_{1}+\beta_{21}\left|\varphi_{1}\left(\vec{r}, t_{k}\right)\right|^{2}\right. \\
\left.+\beta_{22}\left|\varphi_{2}\left(\vec{r}, t_{k}\right)\right|^{2}\right] \varphi_{2}(\vec{r}, t)
\end{gathered}
$$

which can be solved exactly. Integrating (19) from $t_{k}$ to $t$, one gets

$$
\begin{aligned}
\varphi_{1 j k l}^{(2)}= & \varphi_{1 j k l}^{(1)} \\
& \times e^{-i \tau\left[V_{1}+\left(\beta_{11}-\lambda\right)\left|\varphi_{1}\left(\vec{r}, t_{n}\right)\right|^{2}+\beta_{12}\left|\varphi_{2}\left(\vec{r}, t_{n}\right)\right|^{2}-3 \lambda \partial_{n n}\left(\xi^{n}\right)\right]}, \\
\varphi_{2 j k l}^{(2)}= & \varphi_{2 j k l}^{(1)} \\
& \times e^{-i \tau\left[V_{1}+\left(\beta_{11}-\lambda\right)\left|\varphi_{1}\left(\vec{r}, t_{n}\right)\right|^{2}+\beta_{12}\left|\varphi_{2}\left(\vec{r}, t_{n}\right)\right|^{2}-3 \lambda \partial_{n n}\left(\xi^{n}\right)\right]}, \\
& (j, k, l) \in \Omega_{M K L}^{0},
\end{aligned}
$$

where $\left.\partial_{n n} \xi^{n}(r)\right|_{j k l}=-\sum_{p q s}\left(u_{p}^{2}+u_{q}^{2}+u_{s}^{2}\right) \gamma_{p q s} \sin (j p \pi / M)$ $\sin (k q \pi / K) \sin (l s \pi / L)$.

The discrete sine transform coefficients of the vector $\left.\phi_{p q s}(r)\right|_{\left(x_{j}, y_{k}, z_{l}\right)}$ for $(p, q, s) \in \Omega_{M K L}$ are

$$
\begin{aligned}
\gamma_{p q s}= & \frac{1}{u_{p}^{2}+u_{q}^{2}+u_{s}^{2}} \frac{8}{M K L} \\
& \times \sum_{j=1}^{M-1} \sum_{k=1}^{K-1} \sum_{l=1}^{L-1}\left|\varphi_{1 j k l}^{(1)}\right|^{2} \sin \left(\frac{j p \pi}{M}\right) \sin \left(\frac{k q \pi}{K}\right) \sin \left(\frac{l s \pi}{L}\right) .
\end{aligned}
$$

Let $\varphi_{1 j k l}^{n}$ and $\varphi_{2 j k l}^{n}$ be the approximations of $\varphi_{1}\left(x_{j}, y_{k}, z_{l}, t_{n}\right)$ and $\varphi_{2}\left(x_{j}, y_{k}, z_{l}, t_{n}\right)$, respectively, which are the solution of (9). For $n=1,2, \ldots$, a second-order time-splitting and sine spectral method for solving (9) via the standard Strang splitting is [14-16]

$$
\begin{gathered}
\varphi_{1 j k l}^{(1)}=\sum_{p=1}^{M-1} \sum_{q=1}^{K-1} \sum_{s=1}^{L-1} e^{-i \tau\left(\left(u_{p}^{2}+u_{q}^{2}+u_{s}^{2}\right) / 4\right)} \alpha_{p q s} \sin \frac{j p \pi}{M} \\
\times \sin \frac{k q \pi}{K} \sin \frac{l s \pi}{L},
\end{gathered}
$$

$$
\begin{aligned}
& \varphi_{2 j k l}^{(1)}=\sum_{p=1}^{M-1} \sum_{q=1}^{K-1} \sum_{s=1}^{L-1} e^{-i \tau\left(\left(u_{p}^{2}+u_{q}^{2}+u_{s}^{2}\right) / 4\right)} \alpha_{p q s}^{\prime} \\
& \quad \times \sin \frac{j p \pi}{M} \sin \frac{k q \pi}{K} \sin \frac{l s \pi}{L}, \\
& \varphi_{1 j k l}^{(2)}=\varphi_{1 j k l}^{(1)} e^{-i \tau\left[V_{1}+\left(\beta_{11}-\lambda\right)\left|\varphi_{1}\left(r, t_{n}\right)\right|^{2}+\beta_{12}\left|\varphi_{2}\left(r, t_{n}\right)\right|^{2}-3 \lambda \partial_{n n}\left(\xi^{n}\right)\right]}, \\
& \varphi_{2 j k l}^{(2)}=\varphi_{2 j k l}^{(1)} e^{-i \tau\left[V_{1}+\left(\beta_{11}-\lambda\right)\left|\varphi_{1}\left(r, t_{n}\right)\right|^{2}+\beta_{12}\left|\varphi_{2}\left(r, t_{n}\right)\right|^{2}-3 \lambda \partial_{n n}\left(\xi^{n}\right)\right]},
\end{aligned}
$$

$$
\begin{aligned}
\varphi_{1 j k l}^{n+1}=\sum_{p=1}^{M-1} \sum_{q=1}^{K-1} \sum_{s=1}^{L-1} e^{-i \tau\left(\left(u_{p}^{2}+u_{q}^{2}+u_{s}^{2}\right) / 4\right)} \beta_{p q s} & \\
& \quad \times \sin \frac{j p \pi}{M} \sin \frac{k q \pi}{K} \sin \frac{l s \pi}{L},
\end{aligned}
$$$$
\varphi_{2 j k l}^{n+1}=\sum_{p=1}^{M-1} \sum_{q=1}^{K-1} \sum_{s=1}^{L-1} e^{-i \tau\left(\left(u_{p}^{2}+u_{q}^{2}+u_{s}^{2}\right) / 4\right)} \beta_{p q s}^{\prime}
$$

$$
\begin{array}{r}
\times \sin \frac{j p \pi}{M} \sin \frac{k q \pi}{K} \sin \frac{l s \pi}{L} \\
(j, k, l) \in \Omega_{M K L}^{0}
\end{array}
$$



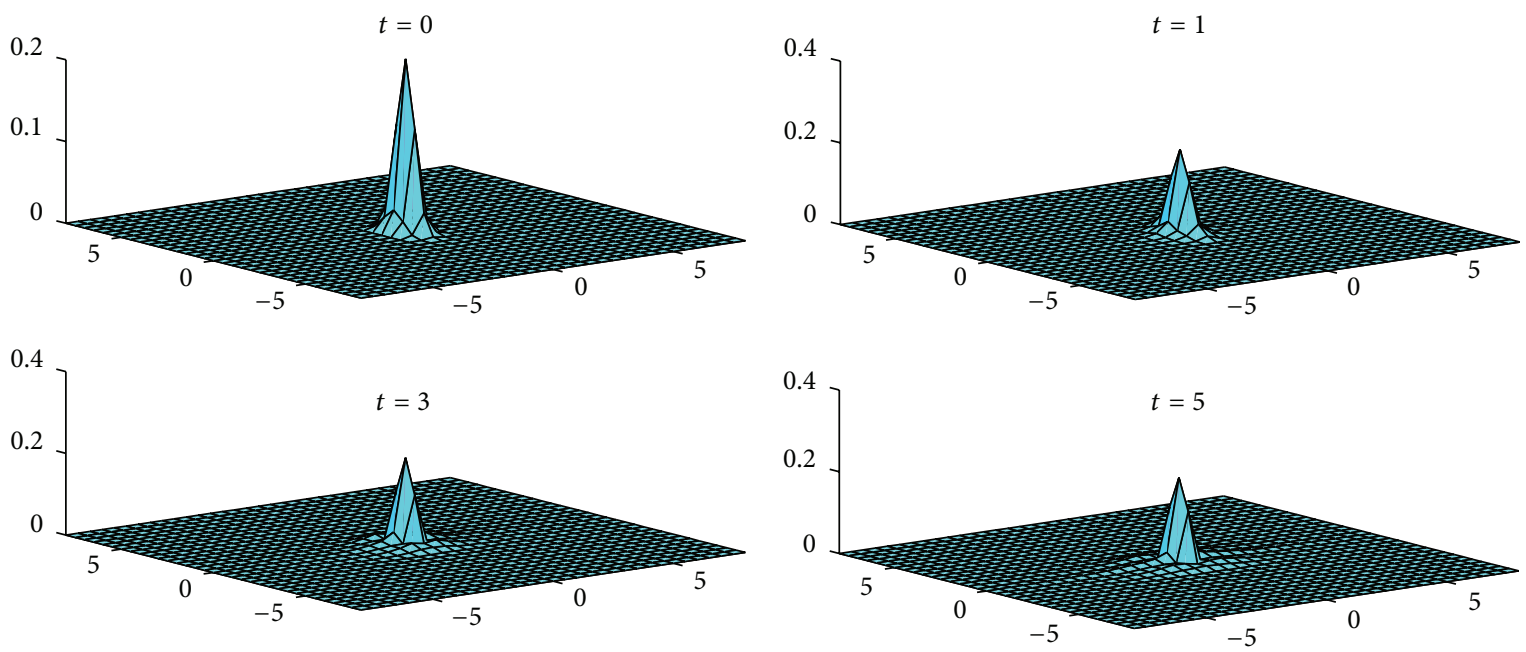

FIGURE 3: The wave function evolution according to time. Surface plots for $\left|\varphi_{2}(x, 0, z, t)\right|^{2}$ at different times.

where

$$
\begin{aligned}
& \beta_{p q s}^{\prime}=\frac{8}{M K L} \sum_{p q s} \varphi_{2 j k l}^{(2)} \sin \frac{j p \pi}{M} \sin \frac{k q \pi}{K} \sin \frac{l s \pi}{L} \\
& \beta_{p q s}=\frac{8}{M K L} \sum_{p q s} \varphi_{1 j k l}^{(2)} \sin \frac{j p \pi}{M} \sin \frac{k q \pi}{K} \sin \frac{l s \pi}{L} .
\end{aligned}
$$

\section{Numerical Results}

3.1. Example with the Same Initial Condition. The confining potential is a cigar potential with $V(r)=(1 / 2)\left(x^{2}+y^{2}+\right.$ $\left.0.04 z^{2}\right)$. Consider the dynamics of the BEC in the cigar trap. The initial condition is given as follows:

$$
\begin{array}{r}
\varphi_{1,0}=\varphi_{1}(x, y, z, 0)=\pi^{-3 / 4} \gamma_{x}^{1 / 2} \gamma_{z}^{1 / 4} e^{-(1 / 2)\left(\gamma_{x}\left(x^{2}+y^{2}\right)+\gamma_{z} z^{2}\right)}, \\
\varphi_{2,0}=\varphi_{2}(x, y, z, 0)=\pi^{-3 / 4} \gamma_{x}^{1 / 2} \gamma_{z}^{1 / 4} e^{-(1 / 2)\left(\gamma_{x}\left(x^{2}+y^{2}\right)+\gamma_{z} z^{2}\right)}, \\
(x, y, z) \in R^{3} .
\end{array}
$$

Here $\gamma_{x}=\omega_{x} / 2 \omega_{0}, \gamma_{z}=\omega_{z} / 2 \omega_{0}$, and $\omega_{0}=\min \left\{\omega_{j x}, \omega_{j y}, \omega_{j z}\right\}$. We solve this system on $[-8,8]^{3}$ with $h_{x}=h_{y}=h_{z}=1 / 2$ and $\Delta t=0.01$.

Figure 1 shows energy evolutions of dipole BEC. And the energy is conserved. Figures 2 and 3 show the wave function evolutions according to time.

3.2. Example with the Different Initial Condition. The confining cigar trap potential is $V(r)=(1 / 2)\left(x^{2}+y^{2}+0.04 z^{2}\right)$. Solve the dynamics problem for a dipolar BEC with the cigar trap. The initial condition is

$$
\begin{aligned}
\varphi_{1,0} & =\varphi_{1}(x, y, z, 0) \\
& =\pi^{-3 / 4} \gamma_{x}^{1 / 4} \gamma_{y}^{1 / 4} \gamma_{z}^{1 / 4} e^{-(1 / 2)\left(\gamma_{1 x} x^{2}+\gamma_{1 y} y^{2}+\gamma_{1 z} z^{2}\right)},
\end{aligned}
$$

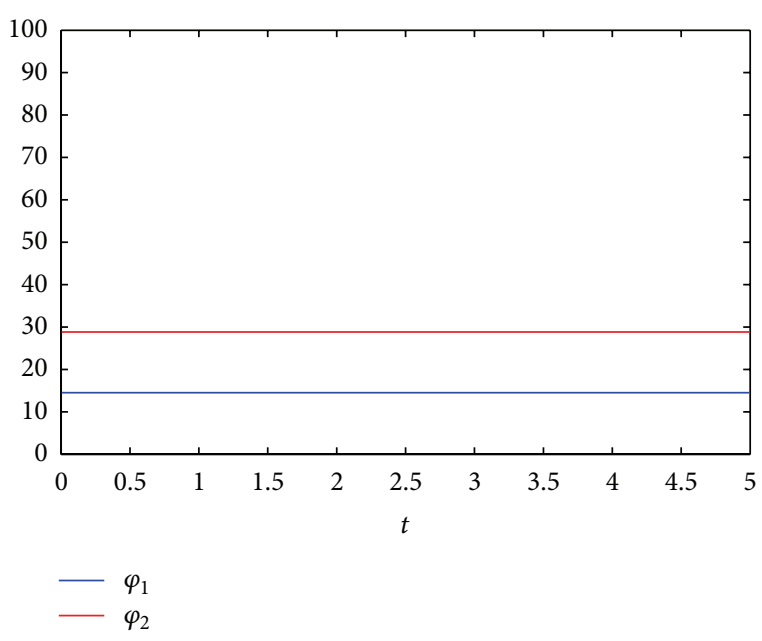

FIgURE 4: The energy evolution according to $t$.

$$
\begin{array}{r}
\varphi_{2,0}=\varphi_{2}(x, y, z, 0)=\frac{1}{2} \pi^{-3 / 8} e^{-(1 / 2)\left(\gamma_{2 x} x^{2}+\gamma_{2 y} y^{2}+\gamma_{2 z} z^{2}\right)}, \\
(x, y, z) \in R^{3} .
\end{array}
$$

Figure 4 shows energy evolutions of dipole BEC. And the energy is conserved. Figures 5 and 6 show the wave function evolutions according to time.

\section{Conclusion}

An efficient numerical method is presented for computing the dynamics of the dipolar Bose-Einstein condensates based on two coupled three-dimensional Gross-Pitaevskii equations where one equation has a dipole-dipole interaction potential and the other one has only the usual s-wave contact interaction. Using equality (5), we can reformulate the GPE for dipolar BEC into a Grosss-Pitaevskii-Poisson type system. Numerical examples are given to show the efficiency of our 

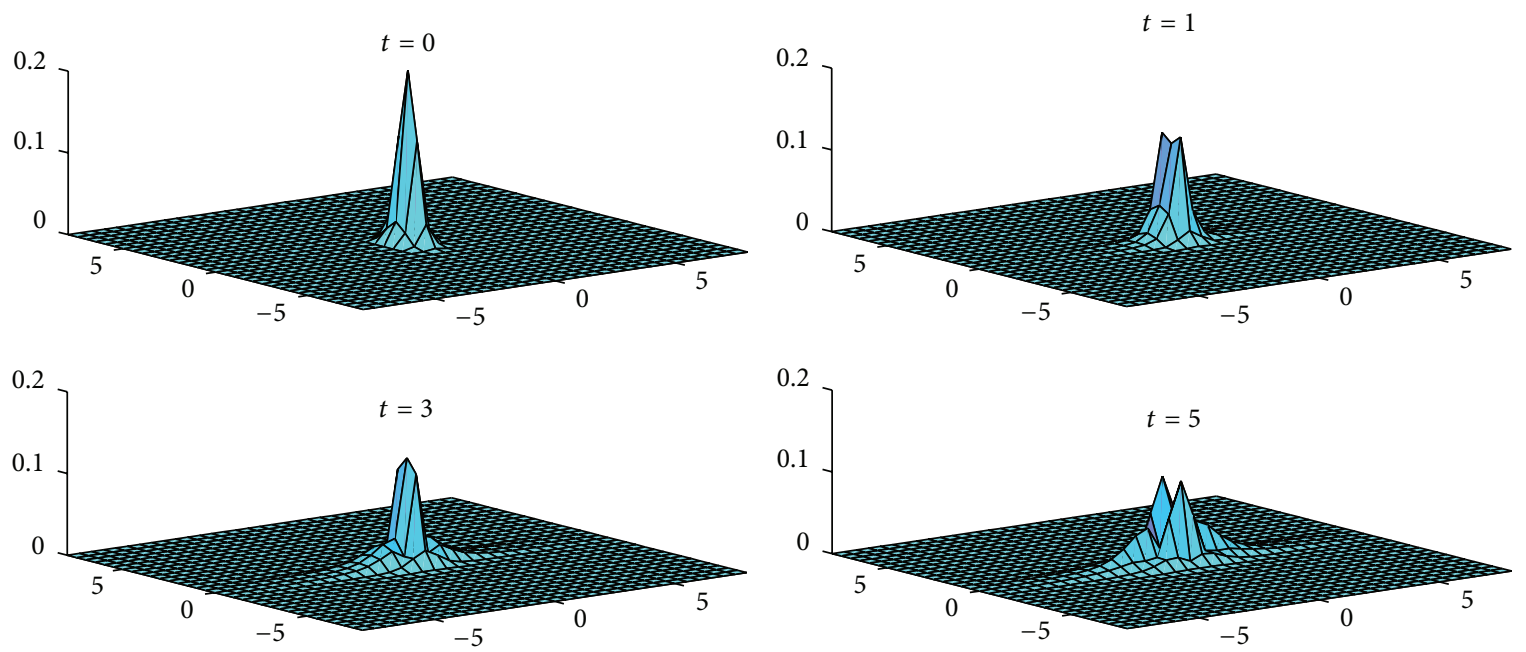

FIGURE 5: The wave function evolution according to time. Surface plots for $\left|\varphi_{1}(x, 0, z, t)\right|^{2}$ at different times.
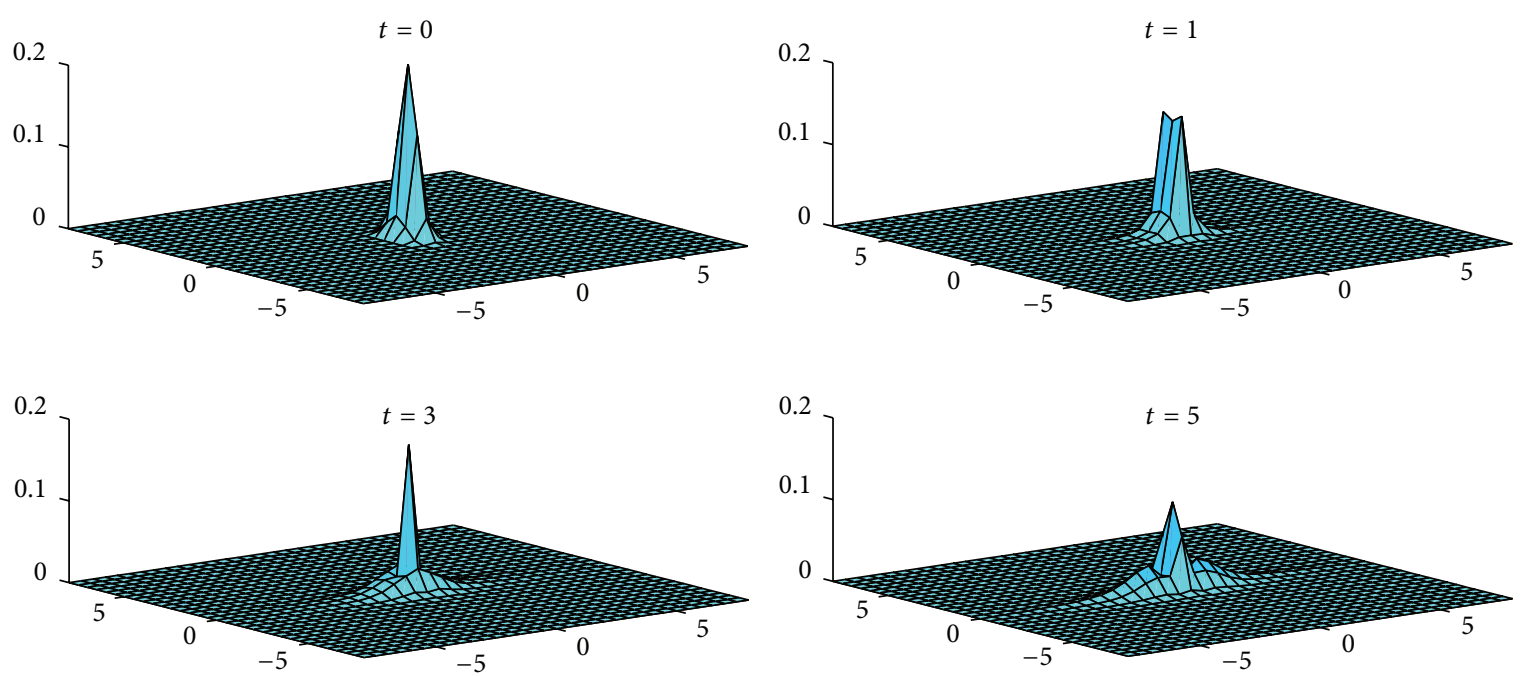

FIGURE 6: The wave function evolution according to time. Surface plots for $\left|\varphi_{2}(x, 0, z, t)\right|^{2}$ at different times.

method. The figures show the evolution of the wave function with time. And in all cases, total energy is conserved. The results agree with the previous work [16]. Numerical results are given to demonstrate the efficiency of our numerical method.

\section{Authors' Contribution}

All authors conceived the study, participated in its design and coordination, drafted the paper, participated in the sequence alignment, and read and approved the final paper.

\section{Acknowledgments}

This work was supported by National Science Foundation of China (no. 11171032) and Beijing Municipal Education Commission (no. KM201110772017).

\section{References}

[1] S. S. ItaevskiiL, Bose-Einstein Condensation, Oxford University, New York, NY, USA, 2003.

[2] A. Griesmaier, J. Werner, S. Hensler, J. Stuhler, and T. Pfau, "Bose-Einstein condensation of chromium," Physical Review Letters, vol. 94, no. 16, Article ID 160401, 2005.

[3] J. Stuhler, A. Griesmaier, T. Koch et al., "Observation of dipoledipole interaction in a degenerate quantum gas," Physical Review Letters, vol. 95, no. 15, Article ID 150406, 2005.

[4] V. Schweikhard, I. Coddington, P. Engels, S. Tung, and E. A. Cornell, "Publisher's note: vortex-lattice dynamics in rotating spinor bose-einstein condensates," Physical Review Letters, vol. 93, no. 22, Article ID 210403, 2004.

[5] K. M. Mertes, J. W. Merrill, R. Carretero-González, D. J. Frantzeskakis, P. G. Kevrekidis, and D. S. Hall, ibid. 99, 190402, 2007.

[6] S. B. Papp, J. M. Pino, and C. E. Wieman, ibid. 101, 040402, 2008. 
[7] S. Yi and L. You, "Trapped atomic condensates with anisotropic interactions," Physical Review A, vol. 61, Article ID 041604, 2000.

[8] S. Yi and L. You, "Trapped condensates of atoms with dipole interactions," Physical Review A, vol. 63, Article ID 053607, 2001.

[9] S. Yi and L. You, "Calibrating dipolar interaction in an atomic condensate," Physical Review Letters, vol. 92, no. 19, Article ID 193201, 2004.

[10] K.-T. Xi, J. Li, and D.-N. Shi, "Phase separation of a two-component dipolar Bose-Einstein condensate in the quasi-one-dimensional and quasi-two-dimensional regime," Physical Review A, vol. 84, no. 1, Article ID 013619, 2011.

[11] C. J. Pethick and H. Smith, Bose-Einstein Condensation in Dilute Gases, Cambridge University Press, Cambridge, UK, 2008.

[12] D.-Y. Hua, X.-G. Li, and J. Zhu, "A mass conserved splitting method for the nonlinear Schrödinger equation," Adance in Difference Equtions, vol. 2012, article 85, 2012.

[13] W. Bao and Y. Cai, "Mathematical theory and numerical methods for Bose-Einstein condensation," Kinetic and Related Models, vol. 6, no. 1, pp. 1-135, 2013.

[14] W. Bao and Y. Zhang, "Dynamics of the group state and central vortex states in Bose-Einstein condensation," Mathematical Models and Methods in Applied Sciences, vol. 15, no. 12, pp. 18631896, 2005.

[15] G. Strang, "On the construction and comparison of difference schemes," SIAM Journal on Numerical Analysis, vol. 5, pp. 506517, 1968.

[16] W. Bao, D. Jaksch, and P. A. Markowich, "Numerical solution of the Gross-Pitaevskii equation for Bose-Einstein condensation," Journal of Computational Physics, vol. 187, no. 1, pp. 318-342, 2003. 


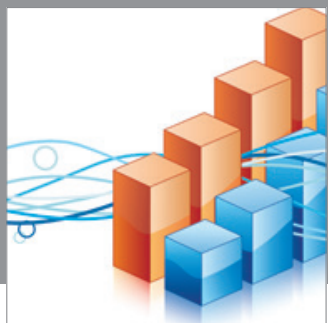

Advances in

Operations Research

mansans

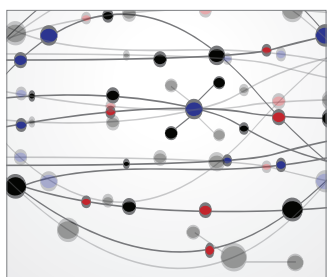

The Scientific World Journal
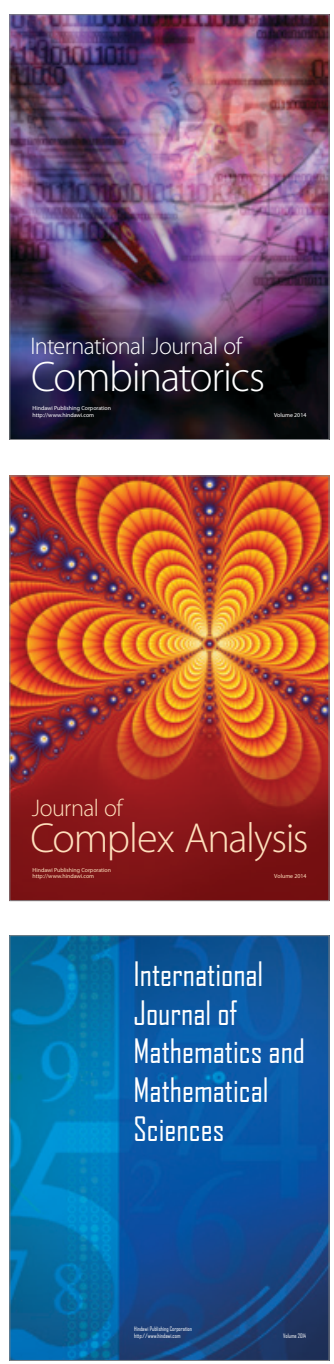
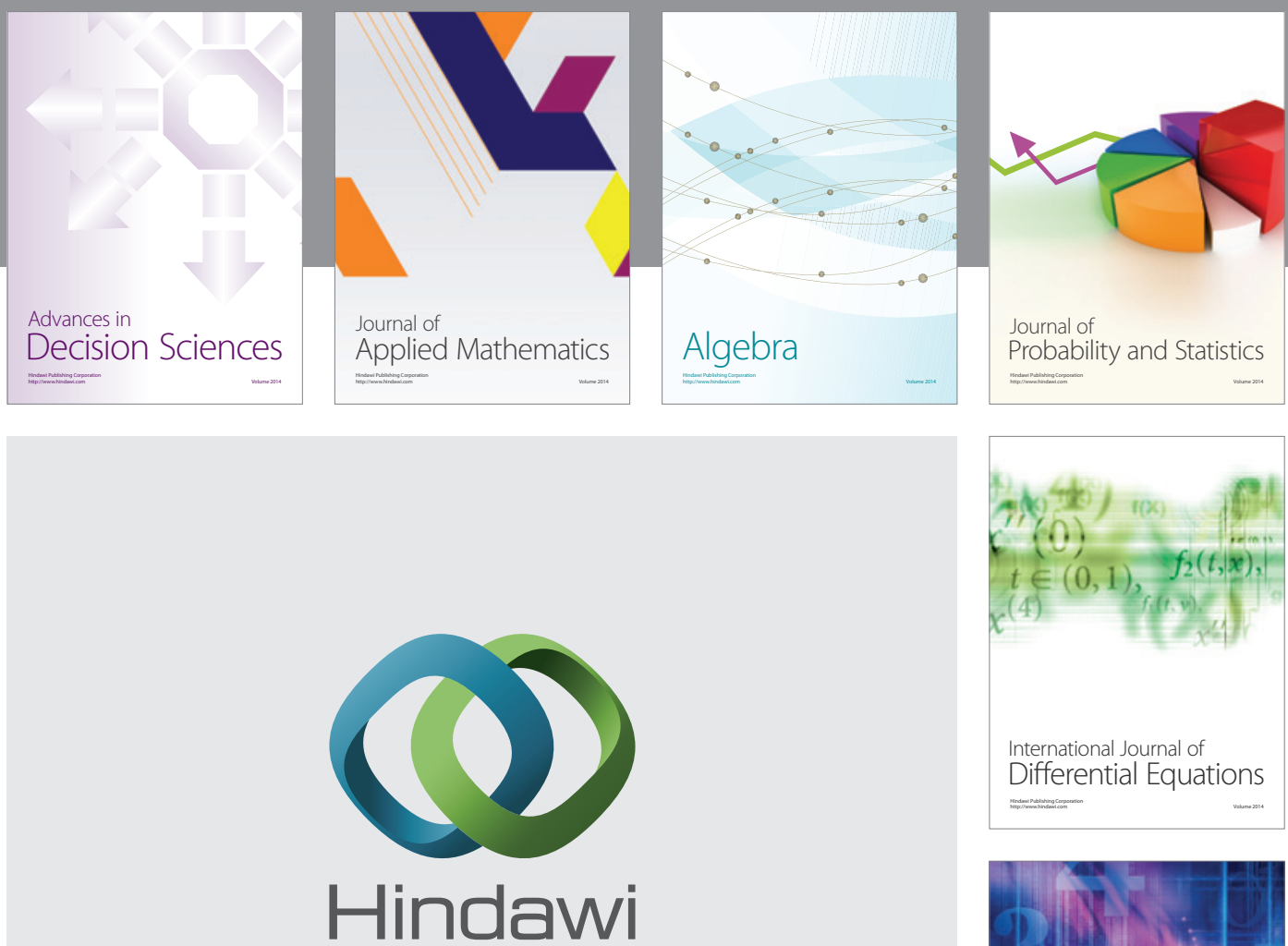

Submit your manuscripts at http://www.hindawi.com
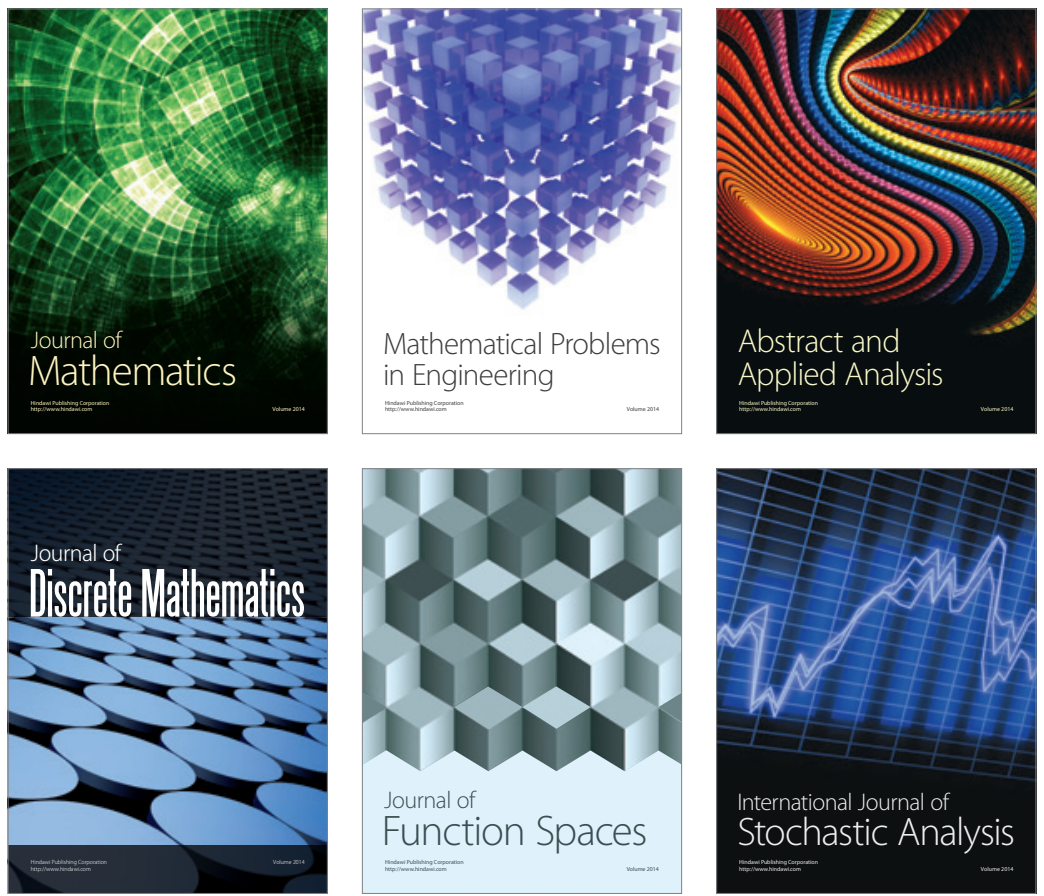

Journal of

Function Spaces

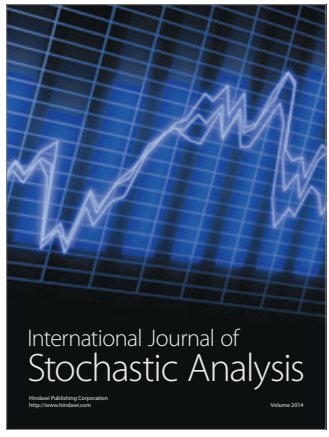

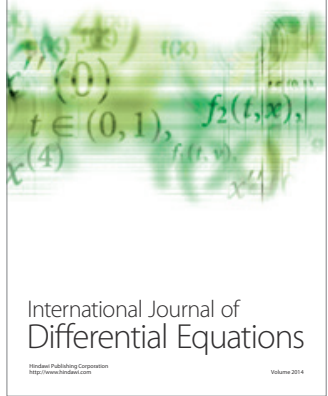
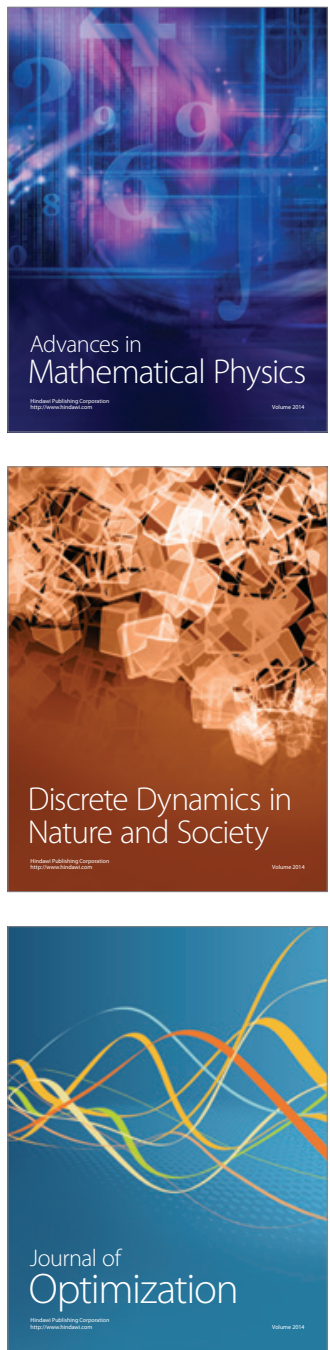